\title{
Synthesis and characterization of chitosan-based polyelectrolyte complexes, doped by quantum dots
}

\author{
N.V. Abuzova*, M.A. Gerasimova, V.V. Slabko, E.A. Slyusareva \\ Siberian Federal University, 79 Svobodny Prospect, 660041 Krasnoyarsk, Russia
}

\begin{abstract}
Doping of polymer particles by a fluorophores results in the sensitization within the visible spectral region becoming very promising materials for sensor applications. Colloids of biocompatible chitosan-based polyelectrolyte complexes (PECs) doped with quantum dots (QD) of CdTe and CdSe/ZnS (with sizes of 2.0-2.4 nm) were synthesized and characterized by scanning electron microscopy, dynamic light scattering, $\zeta$-potential measurements, absorption and luminescence (including time-resolved) spectroscopy. The influence of ionic strength $(0.02-1.5 \mathrm{M})$ on absorption and photoluminescence properties of encapsulated into PEC and unencapsulated quantum dots was investigated. The stability of the emission intensity of the encapsulated quantum dots has been shown to be strongly dependent on concentration of quantum dots.
\end{abstract}

Keywords: quantum dots, polyelectrolyte complex, chitosan, absorption, luminescence, stability

\section{INTRODUCTION}

Quantum dots are widely used for bio-imaging and biosensing applications due to their unique optical properties [1]. However, the most of QD materials are toxic that limits their suitability for application in living organisms. An encapsulation of quantum dots into polymeric structures (both natural and artificial) can be used to reduce toxic load induced by QD dissolution. High stability as well as a high fluorophore content and photoluminescence efficiency of encapsulated QD-systems are prerequisites for their sensing applications.

The natural biopolymer chitosan exhibits an unique adsorption capacity due to its cationic nature in acid media. Chitosan micro- and nanoparticles demonstrate increased mechanical and chemical stability as well as permeability and mobility in comparison with molecular chitosan globules in aqueous solutions. For this reason they are extensively used for various biomedical applications [2]. An electrostatic interaction between polycationic chitosan molecules and anionic species is widely used to produce cross-linked chitosan micro- and nanoparticles [3-5]. The application of anionic biopolymers as counter-ions allows combining biodegradability and biocompatibility of the polyelectrolyte complexes with their highly efficient absorbance of the charged species [6-8].

In the present work the QD-doped polyelectrolyte complexes based on two natural biopolymers (chitosan and chondroitin sulfate) were synthesized and characterized. The doping procedure was optimized for different QD types (CdTe core-type and CdSe/ZnS core-shell type) by adjustment of QD concentration in the reaction medium.

\section{EXPERIMENTAL}

\subsection{Materials}

Hydrophilic (stabilized by a carboxyl group) quantum dots CdSe/ZnS (core-shell) and CdTe (core-type) with the size of 2.0-2.4 $\mathrm{nm}$ and emission maximum at $530 \mathrm{~nm}$ were supplied by PlasmaChem, Germany. Low-viscosity chitosan (aminopolysaccharide, 2-amino-2-deoxy- $\beta$-D-glucan) from shrimp shells and sodium salts of chondroitin sulfate A ( $\beta$ glucuronic acid- $(1 \rightarrow 3)$-N-acetyl- $\beta$-galactosamine-4-sulfate- $(1 \rightarrow 4)$ ) from bovine trachea (Figure 1$)$ were supplied by Sigma-Aldrich.

Acetate buffer at $\mathrm{pH} 5.6$ was used for the preparation of $0.1 \% \mathrm{w} / \mathrm{v}$ chitosan solution. Bidistilled water was used for the preparation of $0.1 \% \mathrm{w} / \mathrm{v}$ chondroitin sulfate and QDs stock solutions. Unbuffered water solution with $\mathrm{pH} 5.2$ was prepared using acetic acid and bidistilled water. The ionic strength was adjusted by addition of $4 \mathrm{M} \mathrm{NaCl}$ water solution.

*sci_box@mail.ru 


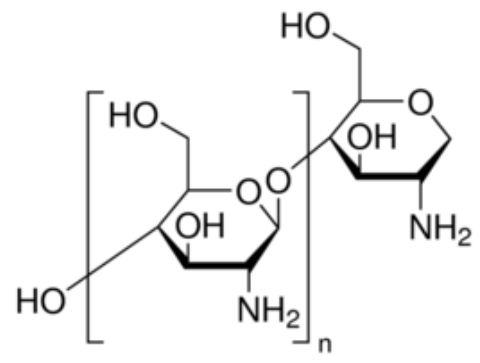

Chitosan

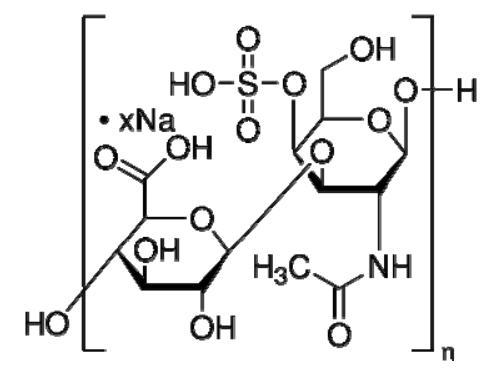

Chondroitin sulfate

Figure 1. Molecular structures of biopolymers used for the synthesis of QD-doped PECs

\subsection{PEC synthesis}

The method used for the synthesis of QD-free PECs from chitosan and chondroitin sulfate was similar to the described previously [8]. Chitosan solution of $0.1 \% \mathrm{w} / \mathrm{v}$ concentration was pre-cleaned from insoluble impurities by filtration through a paper filter. The chondroitin sulfate solution of $0.1 \% \mathrm{w} / \mathrm{v}$ concentration was added dropwise to the chitosan solution under vigorous steering using magnetic stirrer for at least two hours. Volume ratio for polycation (chitosan) and polyanion (chondroitin sulfate) solutions was 2:1. The molecular polymer fraction was separated from the colloid solution using a $5810 \mathrm{R}$ centrifuge (Eppendorf, Germany) at $14,500 \mathrm{rpm}$ and temperature $25^{\circ} \mathrm{C}$ for $5 \mathrm{~min}$. After the elimination of the molecular fraction the precipitate was ultrasonically resuspended in unbuffered solvent with pH 5.2 for $30 \mathrm{~min}$. After the latter procedure the $\mathrm{pH}$-value of the PEC solution grew up to 5.6. Finally, the obtained PEC solution was filtered through a PVDF membrane (Roth, Germany) with $0.45 \mu \mathrm{m}$ pore size. The PEC concentration in the filtrate was estimated taking into consideration the results of a similar synthesis [8] by the value of $\sim 5 \times 10^{-4} \mathrm{~g} \cdot \mathrm{ml}^{-1}$.

To obtain QDs-doped PECs QD stock solution was added to $0.1 \% \mathrm{w} / \mathrm{v}$ chitosan solution in various ratio by volume prior the mixing with the anionic component. The mixture was stirred for $2 \mathrm{~h}$ to achieve the sorption-desorption equilibrium. The latter steps of the synthetic process were the same as for QDs-free PECs. We obtained a three concentration of QDs in PEC after synthesis - high, low and ultralow $\left(10^{-6} \mathrm{M}, 10^{-7} \mathrm{M}, 10^{-8} \mathrm{M}\right.$, respectively).

\subsection{Dynamic light scattering (DLS) and $\zeta$-potential}

The particle size distribution in the investigated PEC solutions was derived from the dynamic light scattering data obtained by three repetitive measurements for each sample using a Zetasizer Nano ZS instrument (Malvern Instruments Ltd., Malvern, UK). The analysis of the autocorrelation function was carried out in approximation of solid spherical particles. $\zeta$-potential measurements were performed on the Delsa Nano instrument (Beckman Coulter, USA); each value represents an average of three repetitive runs.

\subsection{Scanning electron microscopy (SEM)}

The images of PEC particles were taken on a DSM 982 Gemini scanning electron microscope (Zeiss, Germany). Samples were prepared by deposition of a droplet of PEC solution on a silicon stub with subsequent drying.

\subsection{Spectral measurements}

The absorption spectra of QDs in buffer solution were measured on a Lambda 35 UV-Vis spectrophotometer (Perkin Elmer, USA). The photoluminescence (PL) spectra of QDs in buffer solution and those encapsulated into PECs were measured on a Fluorolog 3 spectrofluorimeter (Horiba Jobin Yvon, USA). The photoluminescence was excited at a wavelength of $400 \mathrm{~nm}$. The registered signals were corrected for reabsorption and sensitivity of the registration system.

The time-resolved measurements were performed using the FluoroHub-B timing module (Horiba Jobin Yvon, USA) upon excitation by a pulsed laser diode NanoLED-460 with the excitation maximum at $453 \mathrm{~nm}$ operating at $100 \mathrm{MHz}$ with an optical pulse duration $<1.3 \mathrm{~ns}$. The lifetimes were measured in a maximum of photoluminescence at following wavelengths: $530 \mathrm{~nm}(\mathrm{CdSe} / \mathrm{ZnS}$ QD and CdTe QD in buffer, CdSe/ZnS QD encapsulated into PECs), $545 \mathrm{~nm}$ (CdTe QD encapsulated into PECs). The deconvolution analysis of time-resolved photoluminescence decays were carried out 
using DAS6 software (Horiba). The measured signal was approximated by the sum of three exponential terms. Measurements were carried out using a standard quartz cell $(10 \times 10 \mathrm{~mm})$ at room temperature.

\section{RESULTS}

\subsection{Morphology and $\zeta$-potential of PECs}

Chitosan and chondroitin sulfate globules reveal a polymodal size distribution with the largest ones up to of several microns. The synthesized PECs are represented by spherical loose particles with monomodal size distribution. The data on particle size distribution obtained by both the SEM microscopy (Figure 2) and dynamic light scattering measurements (Z-average size is about $450 \mathrm{~nm}$ with polydispersity index of 0.15 ) are in a good agreement. The $\zeta$-potential of the PECs is positive due to a protonation of free amino groups of chitosan in acid media. The value of $\zeta$-potential is about $+30 \mathrm{mV}$ satisfying the requirement of electrostatic stability of the suspension [7].

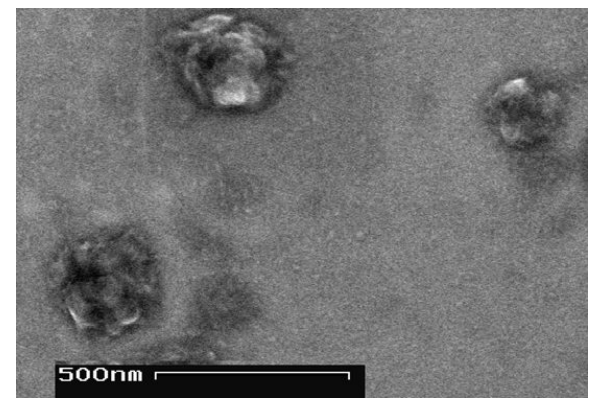

Figure 2. SEM image of QD-doped PECs

\subsection{Absorption and PL properties of free and encapsulated QDs}

The absorption spectra of the QDs in buffer solution (pH 5.6) have an intensive continuous band in UV and VIS region (Figure 3). The PL spectrum represents a well-resolved single peak with the position strongly depending on the QDs size [1]. The emission peaks of CdTe and CdSe/ZnS are at $531 \mathrm{~nm}$ and $525 \mathrm{~nm}$ in buffer solution, respectively. The maxima correspond to QD core size of $2.4 \mathrm{~nm}$ [9] for CdTe core type QDs and $2.0 \mathrm{~nm}[10,11]$ for CdSe/ZnS core-shell type QDs.
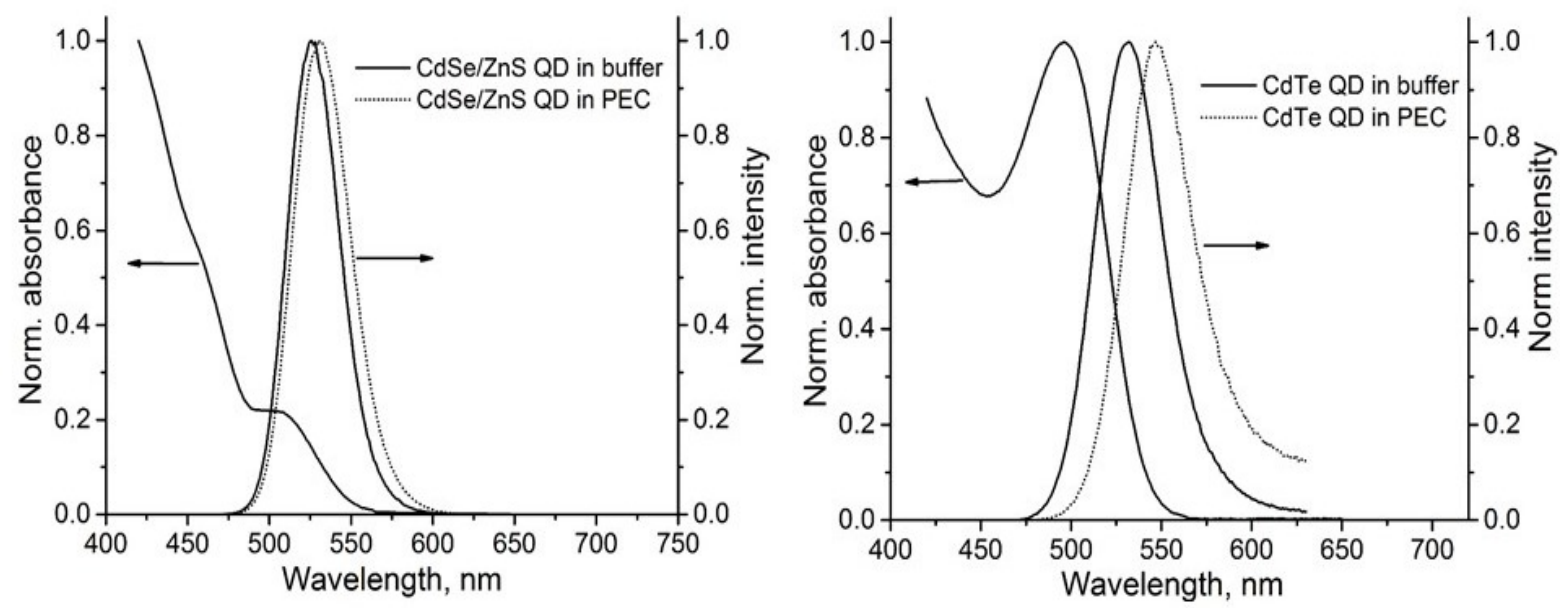

Figure 3. Spectral properties of QD and PL of QD before and after encapsulated in PECs

PL spectra of the encapsulated QDs were found to be shifted (about $4 \mathrm{~nm}$ for core-shell, $14 \mathrm{~nm}$ for core type QDs) towards lower energies in comparison with those of the QDs in buffer solution (Figure 3). This shift is typically attributed to the changes of the QD size in agglomeration process [1]. 
The analysis of the QD photoluminescence decay of demonstrated that the experimental data can be fitted by a sum of three exponentials with different lifetimes. The lifetimes were obtained by averaging of three times considering their amplitude contributions. The average photoluminescence lifetimes of free and PEC-encapsulated QDs are shown in Figure 4. No any pronounced effect of the encapsulation on the PL lifetime was observed. The difference in the lifetime values for two types of QDs (40 ns for CdTe and $13 \mathrm{~ns}$ for $\mathrm{CdSe} / \mathrm{ZnS}$ ) can be attributed to their different core size $[10,11]$ due to the strong dependence of PL lifetimes of QDs on their size [12].

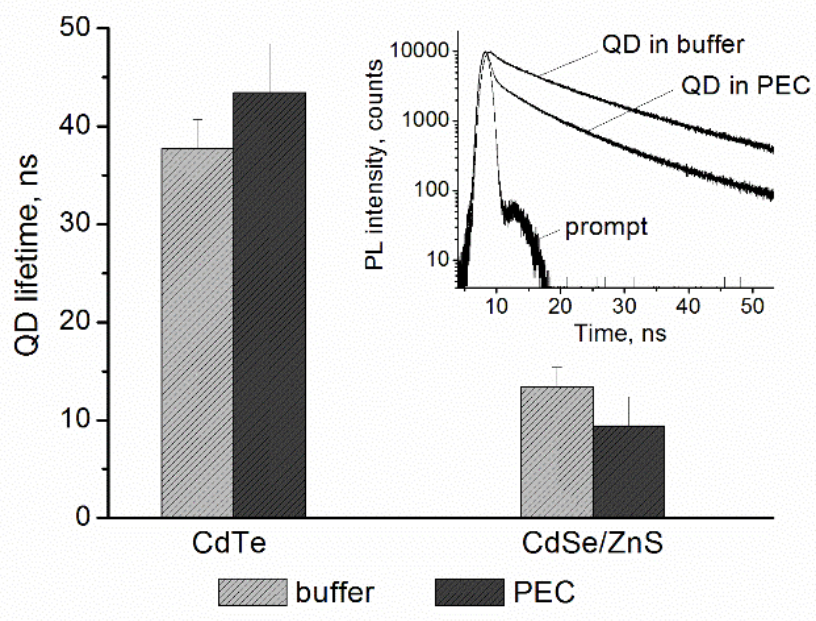

Figure 4. The lifetime of QDs in buffer solution and encapsulated in PEC. Insert shows the example of PL decays of QDs

\subsection{The PL stability of QD-doped PECs}

The emission stability of the QDs encapsulated into PECs was evaluated using the value of PL intensity at maximum of the spectra. Three QD doping solutions with significantly different values of the initial molar concentration of QDs were used during the doping procedure (see the «PEC synthesis»). Encapsulated QDs with a higher concentration demonstrate an increased stability regarding the quenching process: the PL measurements carried out in the course of a week did not reveal any significant quenching of QDs emission (Figure 5). At the same time the PL properties of the samples obtained from solutions with low and ultralow concentrations of QDs undergo noticeable deterioration within the next 1-4 days. Similar results were observed for the both types of the examined quantum dots.
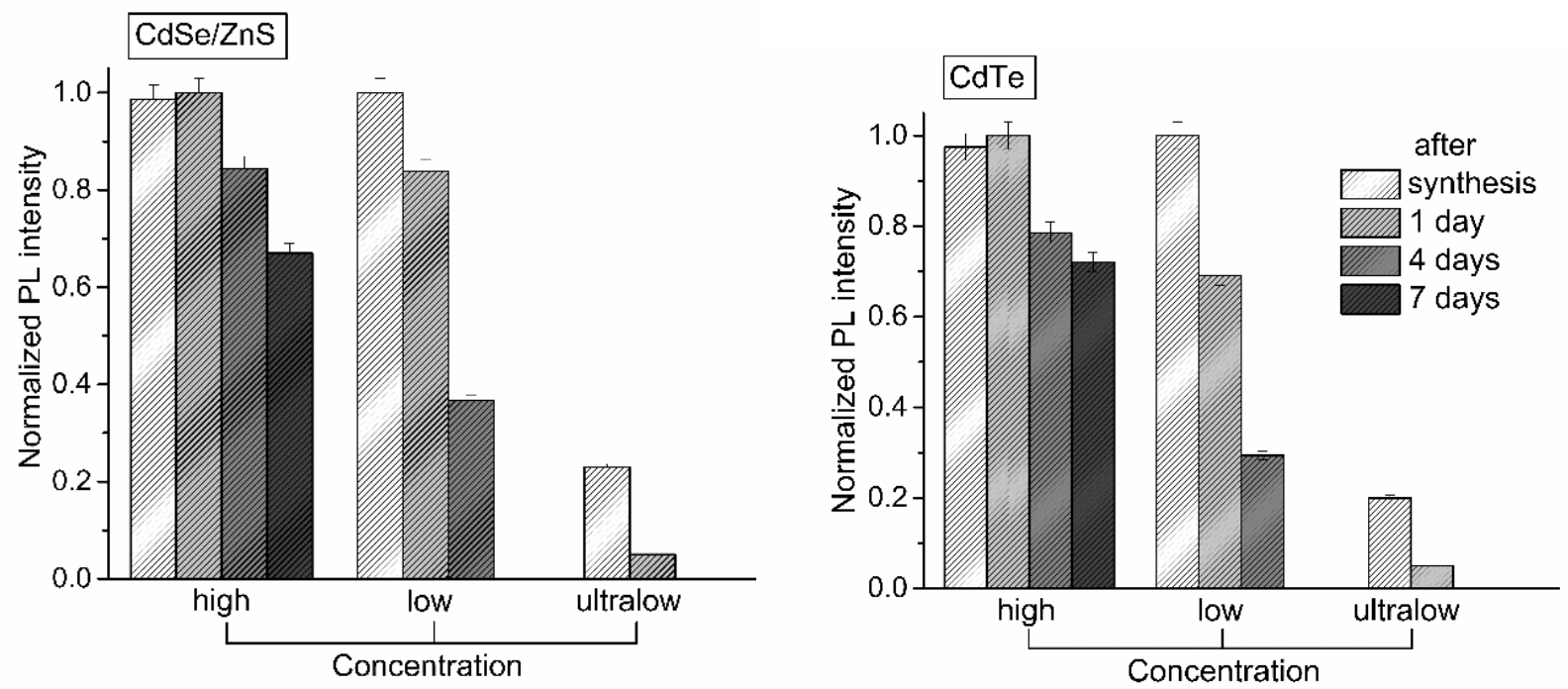

Figure 5. Stability of PL properties of QD-doped PECs 


\subsection{The sensitivity of QD-doped PECs to the solvent ionic strength}

The influence of the ionic strength on the PL intensity of encapsulated quantum dots was investigated (Figure 6). The presence of ions favours the escape of charge carriers or their nonradiative recombination in resulting in the quenching of the PL [1].The effect is more pronounced for the core type QDs. A total PL quenching takes place for CdTe quantum dots at any concentration level if the ionic strange reaches values of $1.5 \mathrm{M}$. The quenching of PL observed for core-shell type QDs at the same experimental conditions amounted about 60\%.

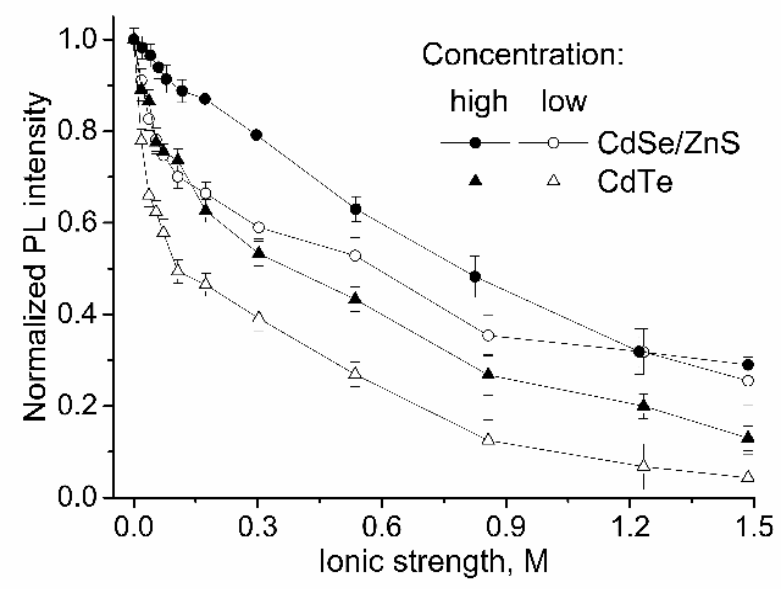

Figure 6. PL dependence of QD-doped PEC on the ionic strength of the solvent

\section{CONCLUSIONS}

Electrostatically stable QDs-doped chitosan-based polyelectrolyte complexes were synthesized for the first time. The photoluminescence lifetime of $\mathrm{CdSe} / \mathrm{ZnS}$ and CdTe quantum dots undergoes no any significant change after their encapsulation into PECs whereas the emission spectral lines are shifted towards the red part of the spectrum. The photoluminescence intensity of the QDs-doped PEC strongly depends on QDs concentrations and ionic strength of the solution. The solvent ionic strength drastically decreases the PL intensity; the effect is more pronounced for lowconcentration solutions of PECs doped by CdTe quantum dots. The emission spectra of the both used QD types demonstrate a high storage stability within a week for the most concentrated $\left(10^{-6} \mathrm{M}\right)$ PEC solution. Taking into consideration the PL lifetime values as well as the storage stability both examined types of quantum dots has potentialities as PEC dopants for further biosensing applications.

\section{ACKNOWLEDGMENTS}

The work is particularly supported by the Ministry of Education and Science of the Russian Federation (No. 3.1749.2014/K, 214/71 for Siberian Federal University) and RFBR, research project No. 14-02-00219 A.

\section{REFERENCES}

[1] Lesnyak, V., Gaponik, N. and Eychmüller, A., "Colloidal semiconductor nanocrystals: the aqueous approach," Chem. Soc. Rev. 42(7), 2905-2929 (2013).

[2] Berger, J., Reist, M., Mayer, J.M., Felt, O. and Gurny, R., "Structure and interactions in chitosan hydrogels formed by complexation or aggregation for biomedical applications," Eur. J. Pharm. Biopharm. 57(1), 35-52 (2004). 
[3] Du, W.L., Xu, Z.R., Han, X.Y., Xu, Y.L. and Miao, Z.G., "Preparation, characterization and adsorption properties of chitosan nanoparticles for eosin Y as a model anionic dye," J. Hazard. Mater. 153(1-2), 152-156 (2008).

[4] Zhao, J. and Wu, J., "Preparation and characterization of the fluorescent release of dye molecule," Chin. J. Anal. Chem. 34, 1555-1559 (2006).

[5] Bhumkar, D.R., Devika, R., Pokharkar, V.B. and Varsha, B., "Studies on effect of pH on cross-linking of chitosan with sodium tripolyphosphate: a technical note," AAPS PharmSciTech. 7(2), E50 (2006).

[6] Liu, Z., Jiao, Y., Wang, Y., Zhou, C. and Zhang, Z., "Polysaccharides-based nanoparticles as drug delivery systems," Adv. Drug Deliv. Rev. 60(15), 1650-1662 (2008).

[7] Boddohi, S., Moore, N., Johnson, P.A. and Kipper, M.J., "Polysaccharide-based polyelectrolyte complex nanoparticles from chitosan, heparin, and hyaluronan," Biomacromolecules 10(6), 1402-1409 (2009).

[8] Slyusareva, E., Gerasimova, M., Plotnikov, A. and Sizykh, A., "Spectral study of fluorone dyes adsorption on chitosan-based polyelectrolyte complexes," J. Colloid Interface Sci. 417, 80-87 (2014).

[9] Yu, W.W., Qu, L., Guo, W. and Peng, X., "Experimental determination of the extinction coefficient of CdTe, CdSe, and CdS nanocrystals," Chem. Mater. 15(14), 2854-2860 (2003).

[10] Mutavdžić, D., Xu, J., Thakur, G., Triulzi, R., Kasas, S., Jeremić, M., Leblanc, R. and Radotić, K., "Determination of the size of quantum dots by fluorescence spectroscopy," Analyst 136, 2391-2396 (2011).

[11] Dabbousi, B.O., Rodriguez-Viejo, J., Mikulec, F.V., Heine, J.R., Mattoussi, H., Ober, R., Jensen, K.F. and Bawendi, M.G., "(CdSe)ZnS core-shell quantum dots: synthesis and characterization of a size series of highly luminescent nanocrystallites," J. Phys. Chem. B 101, 9463-9475 (1997).

[12] Sousa, J.C.L., Ferrari, J.L., Mendonca, C.R. and Schiavon, M.A., "Determination of particle size distribution of water-soluble CdTe quantum dots by optical spectroscopy," RSC Adv. 4(68), 36024-36030 (2014). 